\title{
Development and Application of the Rail-type Inspection Robot used in Substation Rooms
}

\author{
Jiang Keqiang*, Sun Zhizhou, Liu Yongcheng, Sui Jichao, Fu Chongguang, Li Yunchang \\ Shandong Luneng Intelligence Technology Co, LTD., Jinan 250101, China
}

\begin{abstract}
The outdoor inspection robots are widely used in substation at present. At the same time, the requirements of inspection robot used in substation room are put forward. First, the background of substation indoor equipments was analyzed in this paper, and the inspection content requirements of robot used for these equipments were given. Then, the overall structure of intelligent inspection robot was designed. The key components, such as the platform of horizontal movement, motion control system, and PD (Partial Discharge) detection device were closely designed. Finally, the practical application example of inspection robot was given. The application results show that the structure of rail type inspection robot used in substation room is reasonable and its operation is reliable. The inspection robot can work well instead of staff.
\end{abstract}

\section{Preface}

The reliable operation of equipments used in substation room (such as relay protection room、 GIS room、 switchgear room, etc.) is very important for power grid safety. At present, the inspection and operation condition evaluation work of equipments used in substation room is usually finished by the staff $[1,2]$. However, manual inspection and test has some disadvantages such as security risk, low efficiency and high test probability of those equipments no need to be tested together[3]. In order to make the power grid run safely and reliably, the state grid company of China plans to set up strong power grid and intelligent substation[4], and put forward requirements of unmanned inspection and early warning of equipment fault. So it is very important to research and develop rail type inspection robot used for equipments in substation room.

The rail type inspection robot can detect the equipments automatically, show the inspection data and give the potential failure by data analysis. In order to satisfy unmanned inspection requirement, the intelligent inspection robot can be designed together in the design stage of new intelligent substations and set up for the existing common substations.

\section{Introduction of intelligent inspection robot}

\subsection{Functional analysis}

The rail type intelligent inspection robot used in subway station equipment room should have the following functions.

(1)Motion navigation and obstacle avoidance function The layout of the indoor equipments in the substation is different. In order to improve flexibility and adaptability of inspection robot, the track that will be installed on the ceiling or side wall is used to provide the function of support and navigation. The track can be linear, S-type or ring depending on the position of detected equipments.

The lift mechanism which can move together with the detection component on inspection robot body has a large stretch ratio. It can effectively expand the scope of inspection. At the same time, the robot has the obstacle recognition function. The robot can stop running automatically when it detects an obstacle around it and continue to run when the obstacle is removed.

(2)Multiple detection functions

According to the different contents of the inspection, the robot can be equipped with different detecting equipments, such as infrared thermal imager, optical camera and PD (Partial Discharge) detector, which can be used to check the appearance, temperature and partial discharge level of switchgear and other substation indoor equipments.

(3)Comprehensive analysis and early warning function The background system can automatically analyze and deal with different inspection data, for example, it can automatically identify the state of all types of switches, pressure plate, pilot lamp and the value of instruments. At the same time, historical data curves and inspection reports can be automatically generated.

\footnotetext{
Corresponding author: jkq_py@163.com
} 
When the inspection data is greater than the default alert value, the background system will automatically pop up the window for alarm. It also can be used to perform horizontal and vertical comparison of various types of data. Combined with the failure model, the robot can predict some potential faults or hidden dangers of inspected equipments.

(4)Equipment linkage control function

Inspection robots can be used to control some other devices automatically. The indoor lights can be turned on and the automated curtains can be opened by the robot to improve inspection results when then the inspection robot start. The robot can work with the fire automatic alarm system. When a fire occurs, the robot can arrive at the fire position and sent the scene of the fire to control centre automatically.

(5)Auxiliary monitoring function

The inspection robot is equipped with two-way voice intercom equipment, and the auxiliary detection module detects temperature, humidity, ozone and smoke content in real time. These detection contents can be provided to field staff to make accurate judgment for the operation status of substation.

\subsection{System architecture design}

The architecture of indoor rail type intelligent inspection robot system is network distributed, and it includes three parts: the background layer, communication layer and intelligent terminal layer. The terminal layer is located in equipment room, and it is composed of track system, inspection robot body and auxiliary monitoring device. Terminal layer performs inspection tasks and achieve auxiliary monitoring function. The background layer is composed of server, display monitor and intercom equipment, and it is in control of the terminal layer. The background software is used to make the analysis of inspection data.

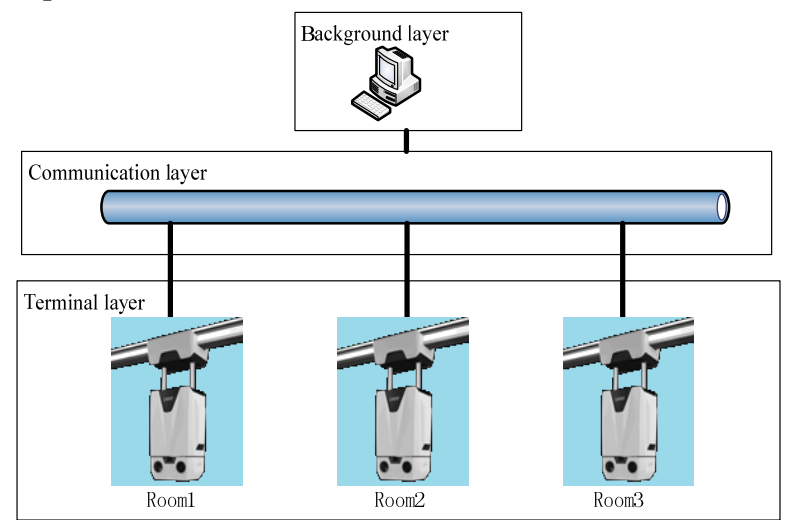

Figure 1. System architecture of inspection robot

Communication layer consists of network switches, photoelectric converters, power line carrier modem and other communications equipment, and it is responsible for the establishment of the network channel between the back-end and intelligent terminal layer. Communication medium between the terminal layer and the background layer can be cable or fiber depending on the distance between the background layer and the terminal layer.
The system supports centralized control mode. Multiple robots can be centrally controlled remotely.
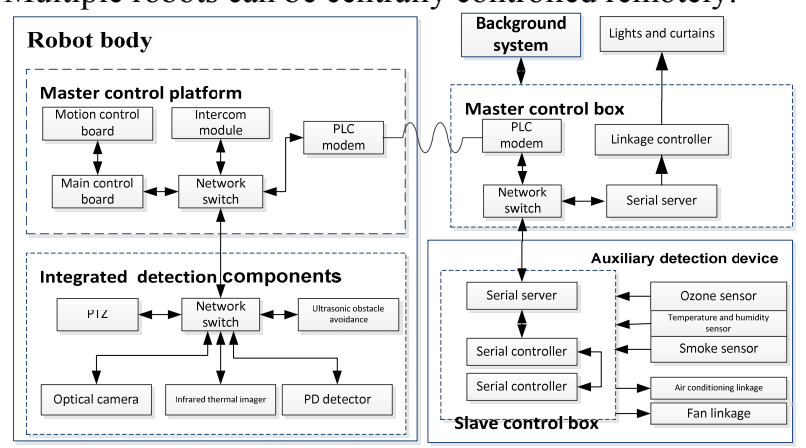

Figure 2. Device structure of inspection robot

\subsection{Robot body design}

Robot movement body moves along aluminum track which is installed on the ceiling or side wall of equipment room. The inspection robot body consists of horizontal sports platform、 master control platform、 lift movement component、PD detection component and PTZ detection component. PTZ detection component consists of optical camera infrared thermal imager and PTZ. PTZ can take these detection equipments achieve horizontal and pitch rotation.

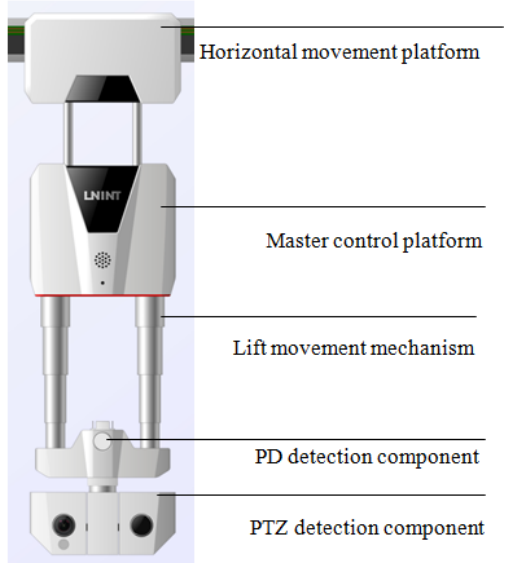

Figure 3. Structure of the robot body

Horizontal movement platform also plays a role of carrier. It is driven by a DC servo motor. The driving force comes from the friction between the wheel installed on horizontal movement platform and the track. The platform can turn around among curved track. The robot is equipped with lifting device which can take detection devices move up and down.

\section{Key technologies of intelligent inspection robot}

\subsection{Design of horizontal turning movement platform}

The detected equipments layout in different rooms is different. In order to expand the scope of the inspection as much as possible, the robot moves along the installed track. The track can be flexibly mounted on the ceiling where the wheeled robot can not reach. The track is used 
to support and navigate the robot, and it acts as the mounting carrier of sliding contact power line and RFID(Radio Frequency Identification). The sliding contact power line is used to provide power for the robot and it act as communication line by power line carrier technique. The RFID is used for motion positioning for motion control.

The track are made of aluminum alloy. The two sides of the upper wing are used to guide, and the upper surfaces of the lower wing are used to support robot. Both sides of the track's rib are used to install other devices such as RFID.

In order to meet environmental and functional requirements, the carrying capacity of the track should be greater than $100 \mathrm{~kg}$ and its life should be greater than 10 years. The design of the track should be easy to be processed and installed and it can be arranged along the s-type. The cross-section of the material would be deformed when bended, so the curve track is made of some machined parts.

The horizontal movement platform is mounted on the upper part of the robot. It is used to support and take all other parts move. There are two bogies on the front and rear ends of the platform, and they can flexibly rotate. There are two guide wheels on the upper and lower side of each bogie. The bogies and guide wheels make the movement of platform smooth.

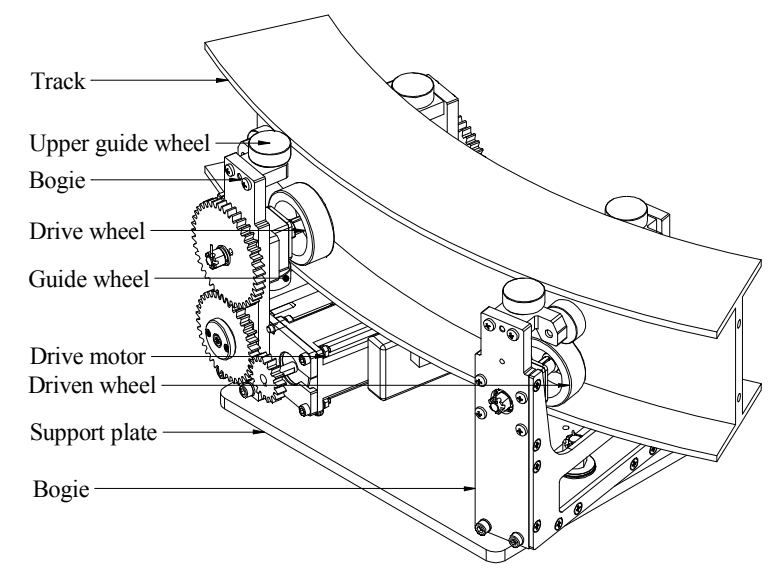

Figure 4. Structure design of horizontal movement platform

Two drive wheels are mounted on both sides of the front bogie, and two driven wheels are mounted on both sides of the rear bogie. The driving force of the robot movement comes from the friction between the driving wheel and the track surface. The drive wheel is driven by a DC servo motor.

\subsection{Design of motion control system}

The basic movement of the robot is divided into horizontal movement and lifting movement. In order to meet the precise identification requirements, the positioning error of each direction should be control within $5 \mathrm{~mm}$.

There are two ways chosen to achieve the positioning of horizontal movement. The RFID installed on the side of the track is used for absolute positioning, and the number of pulses emitted by DC servo motor driver is used for incremental positioning. In autorun mode, the location information sent by the host computer contains the RFID position and the number of pulses which are given by the debugging staff in site. This combined positioning method reduced positioning error compared with the relative positioning method, and improved positioning accuracy in the case of cost reduction.

A proximity switch mounted at the top of the lifting mechanism plays a role of the zero position in the vertical direction. The number of pulses emitted by DC servo motor driver is used to make the positioning of vertical movement. The number of pulses is proportional to the lift distance. The lift stroke is $2 \mathrm{~m}$, so the cumulative error of the lift movement is very small and. Vertical positioning error actual measured is less than $2 \mathrm{~mm}$ when the robot runs at the speed of $0.1 \mathrm{~m} / \mathrm{s}$ in the vertical direction.

The inspection robot will call the PTZ preset position after the robot reaches the specified position to complete the detection work, or call the PD detection component to perform the partial discharge detection task.

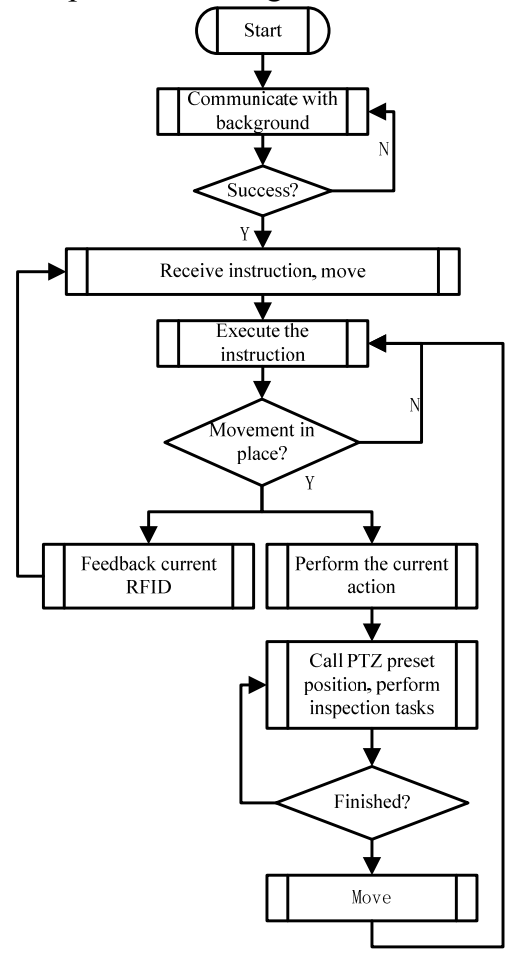

Figure 5. Flow chart of motion control

\subsection{The key technologies of PD detection}

\subsubsection{PD detection component design}

There are two ways used to detect the PD level of the switchgear, named direct measurement method and indirect measurement method[5].

The TEV

( Transient Earth Voltages) measurement method is an indirect measurement method. TEV technology has high detection reliability and sensitivity, so it is very suitable for PD level detection of switchgear[6]. The TEV sensor should be tightly close to the surface of the detected cabinet when the PD is detected. So the PD detection sensor used to carried out PD detection and the method 
used to make TEV sensor close to switchgear should be considered at the same time.

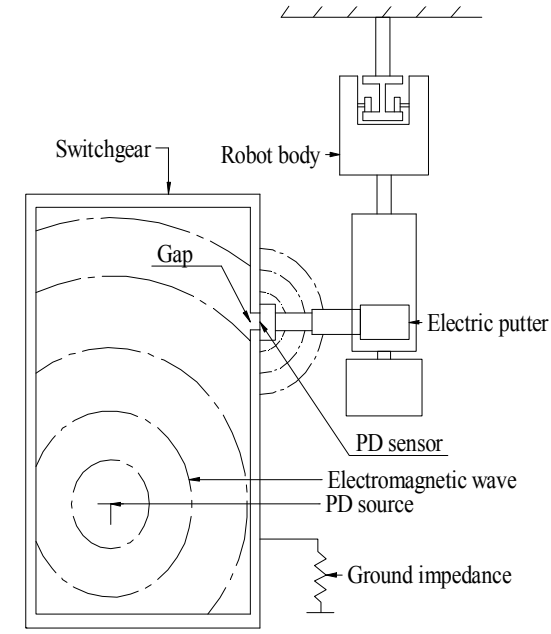

Figure 6. Partial detection diagram

The PD detection component consisted of PD sensor and electric putter is integrated on the robot body. PD sensor can be taken tightly close to the surface of the cabinet by the electric putter which can extend $280 \mathrm{~mm}$. The robot track is installed near the cabinet and the distance between the track and the cabinet should be large enough to ensure barrier-free passage of the robot.

\subsubsection{Data process of $P D$ detection}

PD detection of the switchgear is significantly different from other electrical equipment in terms of the structure and spectral characteristics of the switchgear. First, the discharge part is enclosed in the metal shell, and the sensor of the detection device is difficult to penetrate the inside of the device, so the detection process is difficult to eliminate the influence of environmental electromagnetic noise. Secondly, the switchgear mainly use air insulation or epoxy resin solid insulation, so the dielectric strength is weak, and the electromagnetic discharge spectrum is low, basically with the ambient electromagnetic noise band coincidence, so TEV measurement needs to follow certain process.

The background noise should be detected and recorded before transient voltage detection. The background noise should be measured repeatedly from time to time considering the fluctuation of background noise, then the switchgear transient voltage data is detected following normal process. The detected values of background noise and PD were analyzed synthetically according to certain threshold criteria, and the actual partial discharge data of the switchgear is evaluated finally.

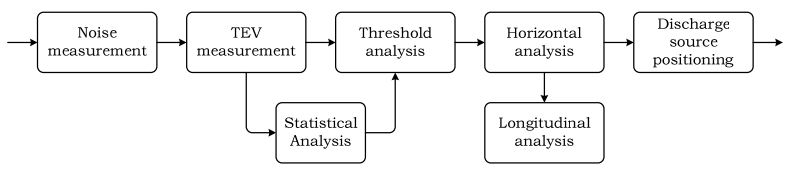

Figure 7. TEV detection data process

There are four methods to analyze the switchgear PD data: threshold analysis, horizontal analysis, longitudinal analysis and statistical analysis. If the simple threshold analysis can not show the information of switchgear PD level, especially when switchgear PD is weak, the horizontal analysis method can be used to judge the PD level of the switchgear by comparing the detection data of the same type of devices.

Compared with the threshold analysis and the lateral analysis technique, the statistical analysis can be used to analyze and discover the development and evolution of the partial discharge state of the switchboard of the power enterprise from the macroscopic point of view, and it can help the operation and maintenance staff to formulate the correct maintenance strategy.

Longitudinal analysis is a kind of analysis method than can be used to analyze the data of the same device at different time. By comparing the data at different times, it can help the staff find the potential defects of the equipments.

\section{Field application}

The robot started work in April 2017, and it has been running for more than six months and got a lot of data. The inspection data were analyzed by horizontal and longitudinal as follow.

Four detected points, two named point 1-1 and 1-2 on No.1 switchgear and the other two named point 2-1 and 2-2 on No.2 switchgear were selected. The No.1 and No.2 switchgears are the same type cabinet. The point 11 and point 2-1 are in the same position on No.1 and No.2 switchgears, and the point 1-2 and 2-2 are the same. The detection data of the four points were analyzed and following analysis results were obtained.

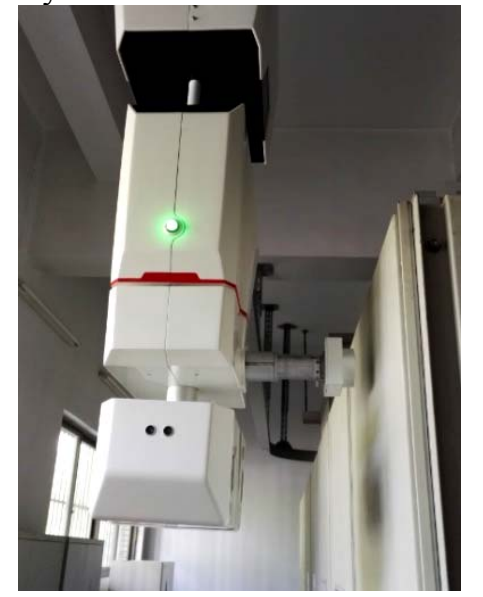

Figure 8. Robot application picture

Point 1-1 and 2-1 are located on the outer upper surfaces of the two switchgears. The PD amplitude of upper part was generally below $15 \mathrm{~dB}$ through horizontal comparative analysis. It showed that the PD level is weak, and the two switchgears run properly. Points 1-2 and 2-2 are located on the back of the bottom of the switchgears, where the cable connectors are inside. The PD level of points 1-2 and 2-2 was higher than that of the points 1-1 and 2-1, and individual data exceeded $20 \mathrm{~dB}$. 


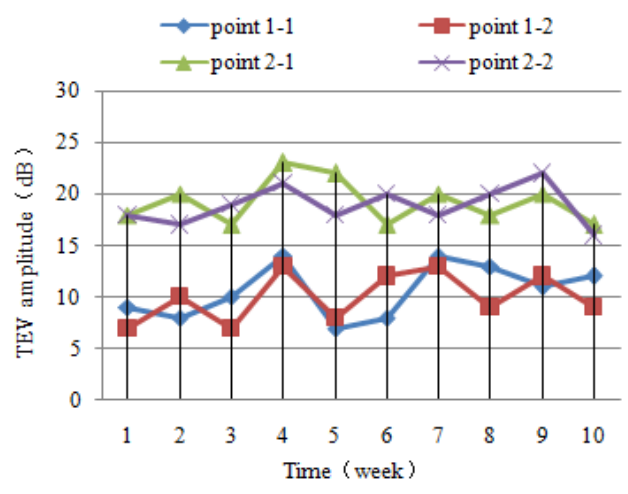

Figure 9. History Curve of PD Detection Data

The PD level of the same point at different time is relatively stable through longitudinal analysis. It showed that the two switchgears run stably and PD level was weak. But the PD amplitude of both two switchgears was bigger than $20 \mathrm{~dB}$ at some same time. Relative humidity information at the same time was analyzed, and it showed that the PD level was generally high when the relative humidity was high. So the relative humidity of the air affects the PD level of the equipments. Comprehensive comparative analysis is helpful for the operation and maintenance staff to make accurate judgment.

\section{Conclusions}

Intelligent inspection means should be used to improve the intelligent level of substation, and rail type inspection robot can be used to realize the automatic inspection of the indoor equipment. Field application shows that the rail type inspection robot used in substation room is reliable. It can work without interruption and the inspection data is more objective and real. So the inspection robot is significant to improve the intelligent level of substation and ensure the safe operation of power equipment. Next the operation function of the robot could be researched in order to expand the scope of robot application and serve the power operation and maintenance better.

\section{References}

1. Cao nan, Li Gang, et al. Key technologies and construction methods of smart substation[J].Power system protection and control, 2011,39(5):63-68

2. Zhou Lihui, Zhang Yongsheng, et al. Development and Application of Equipment Inspection Robot for Smart Substations[J].Automation of Electric Power Systems, 2011,35(19):85-88

3. Lu Shouyin, Qian Qinglin, et al. Development of a Mobile Robot for substation Equipment Inspection[J]. Automation of Electric Power Systems, 2006(13):94-98

4. State Grid. Q/GDW 1168-2013 Regulations of condition-based maintenance \& test for electric equipment[S].Beijing: China electric power press, 2014
5. Li Haide. Research on partial discharge detection methods of HV switchgear based on TEV and Ultrasonic wave[D]. North China Electric Power University,2013.3

6. Ren Ming, Peng Huadong, Chen Xiaoqing, et al. Comprehensive Detection of Partial Discharge in Switchgear Using TEV[J]. HIGH VOLTAGE ENGINEERING,2010,36(10):2460-2466 